\title{
THE REAL AND COMPLEX LORENZ EQUATIONS AND THEIR RELEVANCE TO PHYSICAL SYSTEMS
}

\author{
A.C. FOWLER \\ Department of Mathematics, Massachusetts Institute of Technology, Cambridge, MA 02139, USA \\ J.D. GIBBON* \\ Center for Nonlinear Studies, Los Alamos National Laboratory, Los Alamos, NM 87545, USA \\ and \\ M.J. McGUINNESS \\ Department of Applied Mathematics, California Institute of Technology, Pasadena, CA 91125, USA
}

\begin{abstract}
We summarize some recently obtained results on real and complex Lorenz equations and discuss their possible significance in relation to real fluid dynamical processes.
\end{abstract}

\section{Why Lorenz?}

Two seminal papers (Lorenz [41], Ruelle and Takens [65]) in the recent development of the study of aperiodic motions in dynamical systems both proceeded from a motivation suggested by the phenomenon of turbulence in fluid mechanics. Indeed, 'turbulence' is often referred to in recent dynamical studies, and presumably, much of the continuing interest in such chaotic and aperiodic trajectories in dynamical systems stems from the hope that new and exciting results will provide an impetus and a methodology for understanding old and long-standing problems in the theory of turbulence.

To some extent, it is clear that this has already happened: the phenomena of period doubling (May [47], Feigenbaum [14] and Collet and Eckmann [7]), intermittency (in the sense of Manneville and Pomeau [43]), and hysteresis involving multiple stable states, have been widely observed in

\footnotetext{
* On leave of absence May-August 1982. Permanent address; Department of Mathematics, Imperial College, Queen's Gate, London SW7 2BZ, UK.
}

difference equations (above references; also Tresser et al. [69]), differential equations (Robbins [61], Fraser and Kapral [22], Franceschini and Tebaldi [21]), differential-delay equations (May [48], Hopf et al. [34]); they are also constituent parts of various experimentally observed routes to turbulence in convective and Taylor-Couette flows, as reviewed by Ott [51], Eckmann [13], and Swinney (this volume) and reported by many authors, for example Libchaber and Maurer [38, 39], Libchaber et al. [40], Maurer and Libchaber [45, 46], Fenstermacher et al. [15], Di Prima and Swinney [10], Gollub and Benson [28]. Other 'real-life' systems exhibiting the same sort of chaotic behaviour are Nicholson's blowfly populations (Gurney et al. [29]), white blood-cell populations and the respiratory system (Mackey and Glass [42]), chemical oscillations in the Belousov reaction (Rössler and Wegmann [64]) and the irregular reversals of the earth's magnetic field (Robbins [59, 60]), and at least some of these can (at least in principle) be realistically modelled by a set of ordinary differential equations.

However, the wealth of phenomena and of sys- 
tems to which they apply (both model and experimental) obscures the fact that certain dominant and enduring concerns of fluid dynamicists, that is, the study of transition and turbulence in shear flows (flat plate, pipe flow, Couette flow, Poiseuille flow) and in convection in an unbounded layer, have yielded relatively little to these new techniques; consequently, turbulence of these types has received scant attention in the dynamical systems literature. Other experiments (Taylor-Couette flow, rotating annulus) yield results which can be interpreted by dynamical systems resuits, but not predicted. Our purpose in this paper, therefore, is to outline some possible ways in which the study of simple dynamical systems, and in particular the Lorenz equations (and a complex generalisation thereof) may have some bearing on certain situations in fluid experiments where some kind of 'turbulence' is observed.

Although the equations which bear his name were 'derived' (via truncation) from a model of two-dimensional convection, Lorenz was not interested in convection per se, but in the possible deterministic aperiodicity of atmospheric motions. It is thus remarkable that in the study of idealised models of baroclinic instability which are designed to simulate certain features of the atmospheric circulation, the Lorenz equations have reappeared, but now being derived in a formal manner by the method of multiple scales in situations where the basic flow is marginally unstable.

Baroclinic instability in the atmosphere occurs when an equilibrium state can be maintained in which surfaces of constant density are not parallel to surfaces of constant gravitational potential. Some potential energy is available to be converted into kinetic energy of fluid motions. If small disturbances can grow at the expense of available potential energy, the fluid is said to be baroclinically unstable. The simplest theoretical and experimental model which displays baroclinic instability is the so-called two-layer model which consists of two immiscible fluids, the lighter overlying the heavier and in relative motion, which are rapidly rotated about their vertical axis. Instability occurs at some critical shear in both the inviscid and the viscous cases although the neutral curves differ in each case. For the inviscid case, Pedlosky $[53,55]$ calculated the amplitude equations in the neighbourhood of the critical point and found that the amplitude of a disturbance $A(X, T)$ modulating a carrier wave was governed by the equations

$$
\begin{aligned}
& \left(\frac{\partial}{\partial T}+c_{1} \frac{\partial}{\partial X}\right)\left(\frac{\partial}{\partial T}+c_{2} \frac{\partial}{\partial X}\right) A=\alpha A-\beta A B \\
& \left(\frac{\partial}{\partial T}+c_{2} \frac{\partial}{\partial X}\right) B=\left(\frac{\partial}{\partial T}+c_{1} \frac{\partial}{\partial X}\right)|A|^{2} .
\end{aligned}
$$

These equations were subsequently shown to be a completely integrable system by Gibbon et al. [23] by use of the inverse scattering method. It is easily shown that the sine-Gordon equation is embedded in eqs. (1a) and ( $1 \mathrm{~b}$ ) when the amplitude function $A$ has no phase variation. Define

$$
A=(2 \beta)^{-1 / 2}\left(\frac{\partial}{\partial T}+c_{1} \frac{\partial}{\partial X}\right) \Phi, B=\alpha(1-\cos \Phi) / \beta
$$

and $(1 \mathrm{a}, \mathrm{b})$ reduces to

$\left(\frac{\partial}{\partial T}+c_{1} \frac{\partial}{\partial X}\right)\left(\frac{\partial}{\partial T}+c_{2} \frac{\partial}{\partial X}\right) \Phi=\alpha \sin \Phi$

Consequently, a form of pendulum behaviour is contained in this model. Similar results have been found by Moroz and Brindley [49] using a continuously stratified model (see Drazin [11, 12]). Gibbon and McGuinness [25] have shown that (1a) and $(\mathrm{lb})$ are typical for a certain class of dispersive unstable systems in general, one further example being the self-induced transparency equations of optical pulse propagation.

The connection with the Lorenz model comes when one wants to consider a weakly viscous model instead of the purely inviscid case. This limit has been studied by various authors, the first being Pedlosky [54, 56] followed by Hart [32], although at the time it was not recognised that Lorenz type 
equations had been produced. This was later realised by Pedlosky and Frenzen [57] and Gibbon and McGuinness [24]. Again there is a connection with optics, since it was Haken [31] who showed that the laser equations (which can be expressed in the form of eqs. (1a) and (1b) when damping is excluded) can be transformed into the Lorenz equations when spatial scales are excluded. How the Lorenz equations occur in all these examples as a class (see Gibbon and McGuinness [24, 26] can most easily be described intuitively by considering eqs. (la) and $(1 b)$, in the spatially independent case when weak viscosity (i.e. damping) is added. This is done by the phenomenological introduction of weak viscosity (specifically viscous term $\sim$ amplitude perturbation). This is a distinguished limit which links a purely dispersive to a purely dissipative instability. It can be seen intuitively that the class of examples governed by $(1 \mathrm{a}, \mathrm{b})$ in the purely dispersive limit, become

$$
\begin{aligned}
& \frac{\mathrm{d}^{2} A}{\mathrm{~d} T^{2}}+\Delta_{1} \frac{\mathrm{d} A}{\mathrm{~d} T}=\alpha A-\beta A B, \\
& \frac{\mathrm{d} B}{\mathrm{~d} T}+\Delta_{2} B=\frac{\mathrm{d}}{\mathrm{d} T}\left(|A|^{2}\right)+\Delta_{3}|A|^{2},
\end{aligned}
$$

when weakly viscous terms are added. $\Delta_{1}$ and $\alpha$ are complex numbers if weak dispersive effects are also added (e.g. weak beta plane in the two layer model). A similar form of eqs. (2) has been found by Moroz and Brindley [49] for the continuously stratified model of baroclinic instability.

Under the transformations

$$
\begin{aligned}
& t=\Omega T, \quad \Omega=\operatorname{Re}\left(\Delta_{1}\right)-\frac{1}{2} \Delta_{3}, \\
& X=(2 \beta)^{1 / 2} \Omega^{-1} A, \\
& Z=2 \beta \Omega^{-1} \Delta_{3}^{-1},
\end{aligned}
$$

eqs. (2) can be rewritten as (Fowler et al. [20], Gibbon and McGuinness [26], Gibbon [27])

$$
\begin{aligned}
& \dot{X}=-\sigma X+\sigma Y, \\
& \dot{Y}=(r-Z) X-a Y, \\
& \dot{Z}=\frac{1}{2}\left(X Y^{*}+X^{*} Y\right)-b Z,
\end{aligned}
$$

where $r=r_{1}+\mathrm{i} r_{2}, a=1-\mathrm{i} e, \dot{X} \equiv \mathrm{d} X / \mathrm{d} t,{ }^{*}$ denotes complex conjugate, and

$$
\begin{aligned}
& \sigma=\Delta_{3} / 2 \Omega, \quad b=\Delta_{2} / \Omega, \quad r_{1}=1+2 \operatorname{Re}(\alpha) / \Delta_{3} \Omega, \\
& r_{2}=\left[2 \operatorname{Im}(\alpha)+\Delta_{3} \operatorname{Im}\left(\Delta_{1}\right)\right] / \Omega \Delta_{3}, \quad e=-\operatorname{Im}\left(\Delta_{1}\right) / \Omega .
\end{aligned}
$$

For example, a weak $\beta$-effect in the two-layer model produces complex $\Delta_{1}$ and $\alpha$, and so complex $r$ and $a$, real $b$. In such circumstances, we call (4) the complex Lorenz equations. If no weak dispersive effects are present, $r=r_{1}$ is real, $a=1$, and we regain the (real) Lorenz equations. For higher dimensional models (e.g. the baroclinic models with more than one cross-stream mode), one can obtain an infinite set of Lorenz-type equations (Brindley and Moroz [5], Hart [32], Booty et al. [4]).

Thus we see that, at least in some systems, particularly the baroclinic models, one can really expect the behaviour of the Lorenz equations to have some bearing on the system. In the following two sections, we will discuss some recent results on the behaviour of real and complex Lorenz equations, and their possible implication both for baroclinic and other models.

\section{2. 'Real' behaviour}

The behaviour of the real Lorenz equations is well known. As the bifurcation parameter $r$ is increased (dissipation is decreased), the origin bifurcates to two non-trivial steady states, which undergo subcritical Hopf bifurcation at a critical value $r_{\mathrm{c}}$. Above $r_{\mathrm{c}}$, one can see a bewildering variety of motions, including alternating régimes of chaotic and periodic orbits separated by intermittent and period-doubling transitions. In addition (Fowler and McGuinness [18]), hysteresis occurs between periodic and chaotic/periodic solutions. All these phenomena, and a rationale which helps an understanding of them, are detailed in the recent book by Colin Sparrow [67]. Geometrically, one can understand the existence of a 
strange invariant set by the occurrence at some value $r=r_{\mathrm{h}}$ of a homoclinic orbit (that is, an orbit which begins and ends at an equilibrium point of the equations). As $r$ passes through $r_{\mathrm{h}}$, a homoclinic 'explosion' takes place which 'produces' an infinite number of periodic and aperiodic orbits. These orbits can only disappear in further homoclinic orbits, period-doubling cascades (reversed) or Hopf bifurcations. Topological considerations imply that there will be many period-doubling windows whose 'function' is to remove orbits: this suggests that it might be useful in any system to concentrate more on the homoclinic events, rather than on the myriad period-doubling events which these sprawn, except insofar as the observation of a period-doubling cascade may indicate the existence somewhere in parameter space of homoclinicity: of course, these remarks may need adjustment in higher dimensions.

Obviously, the weakly nonlinear baroclinic models contain homoclinicity. This also occurs (heteroclinicity, in fact) at a codimension two bifurcation point in double diffusion (Knobloch and Proctor [36], Da Costa et al. [8]) where one might be able to prove the existence of 'chaos' using essentially a theorem of Shilnikov (Arneodo et al. [1]). It is an interesting point that the Lorenz attractor which obtains as a result of the homoclinicity is parametrically unrelated to the sub-critical Hopf bifurcation at $r_{\mathrm{c}}$, which need not even exist at all. Thus, one should view homoclinic bifurcation as a new and separate entity, which must be analysed in its own right. This observation might be interesting in view of the long-standing effort to connect the onset of turbulence in plane Poiseuille flow at $\mathrm{Re} \approx 1000$ with the sub-critical Hopf bifurcation at $\mathrm{Re}=5772$ (e.g. Stewartson and Stuart [68]) by means of a threshold amplitude, although it must be admitted that it is becoming clear that such a threshold does play a part, by virtue of its long (viscous) relaxation time (Orszag and Patera [50]). Similar, less justifiable, efforts for pipe flow (Davey and Nguyen [9]) might be restored using the concept of bifurcation from infinity (Rosenblat and Davis [63]): however, see Patera and Orszag [52].
It is more obviously likely (or plausible) that convection may contain homoclinicity of some kind, particularly in view of the experimental observations of period-doubling already cited. In fact, it is the homoclinicity in the Lorenz equations (a primitive 'model' of convection) which is responsible for the non-monotone difference map relating successive maxima of $Z$. One might ask whether there is any way to analytically tackle the homoclinicity in these equations, and thereby 'predict' the chaotic behaviour by producing a Lorenz map analytically. To do this, one needs some help, and it turns out this is available in the form of a region in parameter space where $r$ and $\sigma$ are both large (and comparable). In this case the solutions behave relaxationally, as shown in fig. 1 , consisting (for $X$ and $Y$ ) of a series of spikes interspersed with flat 'quiet' periods. In the limit $r / \sigma \sim \mathcal{O}(1)$, $b / \sigma \ll 1$, one can use asymptotic techniques to 'solve' the equations, and produce a difference
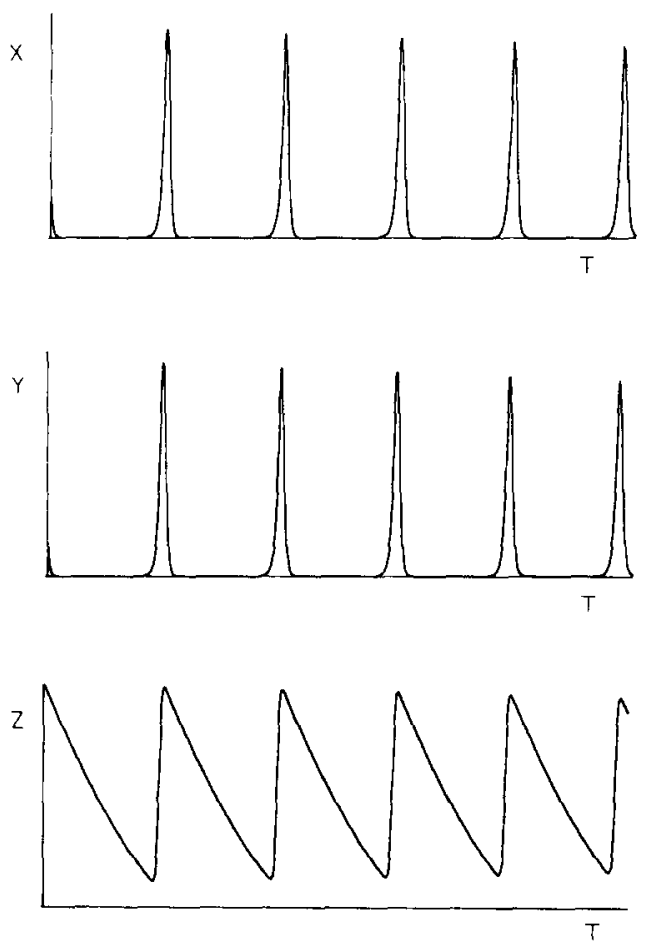

Fig. 1. Relaxational behaviour of $X, Y$ and $Z$ versus time in the real Lorenz equations at parameter values $r=240$, $\sigma=300, b=1$. 


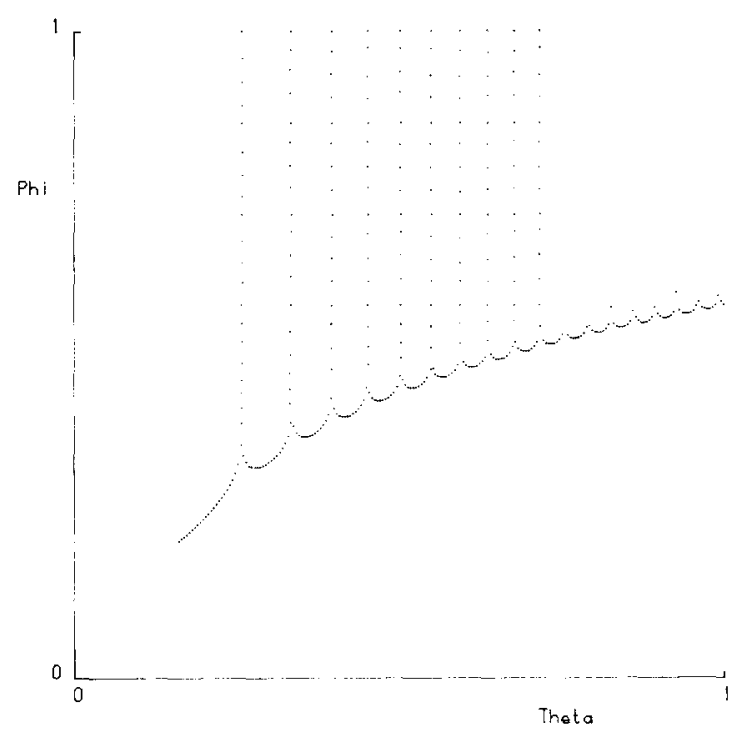

Fig. 2. Difference map for high $r$ and $\sigma(r=160, \sigma=100$, $b=1$ ) relating successive maxima of $Z$ in the real Lorenz equations. Here the $n$th maximum is $Z \approx r[1+\theta]$; the $(n+1)$ th is $Z \approx r[1+\phi]$ and the curve is plotted from the analytic results of Fowler and McGuinness [17]: the first ten cusps are indicated.

map, such as shown in fig. 2 (Fowler and McGuinness [17]). Using this analytic map, we have been able to predict quantitatively the onset of period doubling, and hysteresis (or intermittency) with a fair measure of sucess.

What we wish to draw attention to here, however, is the special pulse-like nature of the solutions. Such pulses occur in many other kinds of system (logistic delay equation, geodynamo, spruce budworm population, jökulhlaups): see Fowler [16] and discussion therein. More germanely, turbulence in shear flows is well known to be intermittent or 'bursty' in character (Maslowe [44]), and an attempt has been made to formalise these observations in an analysis which incorporates two different time scales (Landahl [37]): we recall also the slow viscous time scale of two-dimensional relaxation and the fast advective time scale of three-dimensional instability (Orszag and Pątera [50]).

Virtually the only quasi-analytic theory of convective turbulence at high Rayleigh number is the 'bubble' model of Howard [35], incidentally re- cently much espoused by geophysicists, which has certain experimental support (e.g. Tritton et al. [72]), and seems never to have been experimentally contradicted. In this model, long quiescent conductive phases in which thermal boundary layers grow into an isothermal fluid are interspersed by shortlived, violent convective overturns in which the unstable boundary layers are eradicated. This model requires $\mathrm{Ra}$ (Rayleigh number) and $\sigma$ (Prandtl number) to be large for its validity. The interesting thing is that if we interpret $X, Y$ and $Z$ in the Lorenz equations in terms of their original physical variables (Lorenz [41]), we find that $Z$ is the vertical departure from the conductive temperature profile, $Y$ is the horizontal departure (so producing buoyancy), and $X$ is essentially the velocity. At high $\mathrm{Ra}(r)$ and $\sigma$, we find relaxational motions in the Lorenz equations, in which there are long 'quiet' phases, in which $X \approx 0$ (almost no motion) and $Z$ decays exponentially (thermal boundary layers grow), interspersed with rapid pulses in which $Z$ jumps up, and $X$ becomes significant (convective overturn). In other words, we see Howard's postulated P.D.E. solution in the Lorenz truncation! This obviously suggests a means whereby one might explicitly 'solve' the full convection equations: suffice it to say that this problem has not been satisfactorily resolved as yet. We might also mention that the slow phase is essentially a linear problem, recalling the fast linear three-dimensional instability of Orszag and Patera [50].

What we are tentatively suggesting is that it may be possible to gain a small analytic foothold on the problem of convective turbulence at high $\mathrm{Ra}$ by using direct asymptotic methods on the full equations (and implicitly hoping these equations admit homoclinic solutions), rather than trying to get there through a series of transitions, which apparently precludes any moderately simple explicit computation. One also retains fully nonlinear dynamics in the solutions, and there is no limitation as to being close to any curve in parameter space (in fact, as $\mathrm{Ra}$ and $\sigma \rightarrow \infty$, one is really in a neighborhood of infinity, quite a large area). Such 
an idea is apparently novel in convection (though many authors have studied steady solutions when $\mathrm{Ra} \rightarrow \infty$, e.g. Roberts [62]), but has been touched on in shear flow, where the asymptotic nature of flow disturbances as R (Reynolds number) $\rightarrow \infty$ has long been a consideration in both linear and non-linear studies (Maslowe [44], Benney and Bergeron [3]), as well as in experiments, where the threshold nature of transition has been recognised since the original work of Reynolds [58].

\section{3. 'Complex' behaviour}

When $r$ and $a$ are complex, the set of equations (4) exhibits a notably distinct sequence of bifurcations to those of the real Lorenz equations. Recalling $r=r_{1}+\mathrm{i} r_{2}, a=1-\mathrm{i} e$, we find that for $r_{1}>r_{\mathrm{lc}}=1+\left(e+r_{2}\right)\left(e-\sigma r_{2}\right) /(\sigma+1)^{2}$, the origin is oscillatorily unstable, and a Hopf bifurcation to a limit cycle occurs. Moreover, this limit cycle takes the exact form

$X=A \mathrm{e}^{\mathrm{i} \omega t}, \quad Y=\left[1+\frac{\mathrm{i} \omega}{\sigma}\right] A \mathrm{e}^{\mathrm{i} \omega t}, \quad Z=|A|^{2} / b$,

where

$\omega=\frac{\sigma\left(e+r_{2}\right)}{\sigma+1}, \quad|A|^{2}=b\left(r_{1}-r_{\mathrm{lc}}\right)$.

To find an exact limit cycle in a nonlinear system is remarkable, and worthy of some comment. First, we might hope that a corresponding exact limit cycle might exist in the full equations: for example, in the underlying vorticity equations of the twolayer and continuously stratified models, although this might be modulationally unstable in much the same way as the Benjamin-Feir instability [2] occurs. However, such exact solutions have yet to be found. More realistically, the exact limit cycle in (4) is really due to the fact that when written as a fifth order differential system, the system can be put in the form

$\dot{x}=f(x)$, where $f$ is rotationally invariant, that is, there is a rotation matrix $R(\alpha)$ representing a rotation of the $\boldsymbol{x}$-axes through an angle $\alpha$, such that

$R f(x)=f(R x)$,

and in terms of $R$, the exact limit cycle has the form

$x=R(\omega t) x_{0}$,

where $\boldsymbol{x}_{0}$ is constant. Such a solution is possible, using $R(\alpha) R(\beta)=R(\alpha+\beta)$, if $x_{0}$ and $\omega$ satisfy the nonlinear algebraic eigenvalue problem

$f\left(x_{0}\right)=\omega R^{\prime}(0) x_{0}$.

The rotational symmetry of (9) suggests an analogy with the rotational invariance of the baroclinic models: thus one might hope that (for example) the steady wave régime in rotating annulus experiments (Hide and Mason [33]) might be susceptible to a representation of the form (10), and indeed one might hope for similar results for other rotationally invariant systems, e.g. Taylor vortex flow.

For the complex Lorenz equations, one can do explicit stability analysis of the limit cycle, and we find that a Hopf-like bifurcation to an invariant 2 -torus occurs at a value $r_{1}=r_{\mathrm{lc}}^{\prime}>r_{\mathrm{lc}}$, and the form of the corresponding doubly periodic motion is (in terms of (8))

$\boldsymbol{x}=R(\tilde{\omega} t) \boldsymbol{u}(\tilde{\Omega} t)$,

where $\boldsymbol{u}$ is periodic, and when $\left|r_{1}-r_{\mathrm{ic}}^{\prime}\right| \sim \epsilon^{2}$,

$\tilde{\omega}=\omega+\mathcal{O}\left(\epsilon^{2}\right)$,

$\tilde{\Omega}=\Omega+\mathscr{O}\left(\epsilon^{2}\right)$,

$\boldsymbol{u}=\boldsymbol{x}_{0}+\epsilon\left\{\boldsymbol{u}_{\Omega} \mathrm{e}^{\mathrm{i} \tilde{\Omega} t}+(*)\right\}+\mathcal{O}\left(\epsilon^{2}\right)$

here $\Omega$ is the marginally stable frequency of the perturbation to the limit cycle. Eq. (13) is obtained by using the method of multiple scales, but one may ask whether the special form of (12) extends 
beyond the limited range of (13). It turns out (Fowler and McGuinness [19]) that one can do explicit analysis at $r_{1} \gg 1$ (following Robbins [61], Shimizu [66], Sparrow [67]) which confirms (12) in this limit. Also, spectral analyses at intermediate values all support this form of the torus. Thus, the rotational invariance again gives a special form to the doubly periodic motion, and one might ask if such a form is of relevance to certain rotationally invariant experimental systems.

The bifurcation to a 2-torus in the complex Lorenz equations is numerically found to be subcritical, as its real counterpart is also. (See fig. 3 for a stability map in $r_{1}-r_{2}$ space). As $r_{2}$ is reduced, one observes (numerically) a period-doubling cascade analogous to that in the real Lorenz equations (Robbins [61]): this is easily seen in phase plots of $|X|^{2}$ versus $|Y|^{2}$ for example (which removes the precessing frequency $\bar{\Omega}$ ); we do not know if this cascade is preceded by frequency locking (e.g. Maurer and Libchaber [45], but conjecture that it is the precessing limit cycle $\boldsymbol{u}$ which period-doubles, and we also conjecture that the presence of a perioddoubling torus is due to the occurrence of a

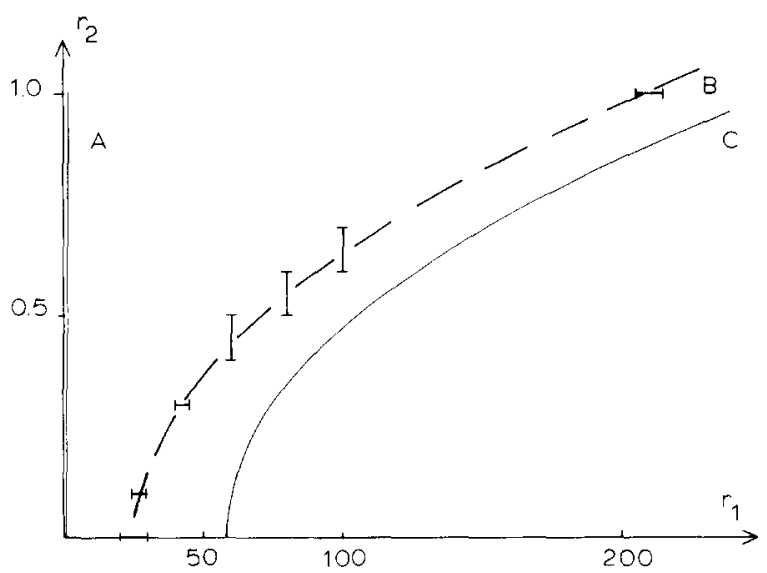

Fig. 3. Parameter space diagram in the $r_{2}$ versus $r_{1}$ plane depicting regions of stability. Between the curves $\mathrm{A}$ and $\mathrm{C}$ the limit cycle is linearly stable $\left(r_{1}<r_{1 c}^{\prime}\right)$. The finite amplitude 2-torus is numerically observed below the approximate dashed curve $\mathrm{B}$ and loses stability by period doubling below $\mathrm{C}$ as $r_{2}$ is reduced. The 'Hopf bifurcation of the limit cycle to a 2-torus on $\mathrm{C}$ is sub-critical, the (unstable) torus existing for $r_{2}$ above C. Here $\sigma=2, b=0.8$ and $e=3 r_{2}$. homoclinic torus at some point in parameter space: this may be suspected on analytic grounds at high $r_{1}$ (Fowler and McGuinness [19]), and probably also at high $r_{1}$ and $\sigma$, although we have not done the corresponding analysis in this case. See also fig. 8 of Fowler et al. [20], where the chaotic torus gets very close to the origin.

Lastly, some comments on scales. In our discussion of the complex Lorenz equations, we have deliberately omitted inclusion of long space derivatives $\partial / \partial X$, as in (1). Apart from simplicity, there is one good reason for this. In many of the real laboratory systems to which we have referred, the geometry is essentially finite in the direction of wave propagation. Thus in the rotating annulus experiments, there is really no $X$ scale near criticality, and purely time-dependent motions should ensue (e.g. amplitude vacillation). It is possible that as the supercriticality ( $A$, say) and the wavenumber increase, there comes a point where (numerically) the circumference is of a length $X \sim \mathcal{O}(1)$. Then the appropriate spatial derivatives might enter, with a requirement of periodicity. It is also plausible (but a guess) that when $\Delta$ thus increases, the first appearance of $X$ dependence is as a bifurcating travelling wave (wave-number vacillation?), as is well known to occur in reaction-diffusion systems as the domain size increases.

Part of the achievement of Pedlosky was to show that the dissipation parameter $r\left(=\mathrm{E}^{1 / 2} / \epsilon\right.$ in the baroclinic problems, where $\mathrm{E}$ is the Ekman number and $\epsilon$ is the Rossby number) defines a distinguished limit when $r \sim|\Delta|^{1 / 2}$. Thus when $r \gg|\Delta|^{1 / 2}$, viscosity dominates, and amplitude equilibration occurs; when $r \ll|\Delta|^{1 / 2}$, one obtains conservative (e.g. sineGordon) equations whose amplitude depends entirely on initial conditions, and thus experimentally will be very susceptible to noise. If $r \sim|\Delta|^{1 / 2}$, one regains the rich structure of the Lorenz equations. When $r \ll|\Delta|^{1 / 2}$, however, conservative waves (e.g. solitons) will evolve over a long viscous time scale, no matter how small $r$ is: in a sense, the viscous time scale is the true scale over which transients die out, and the 'state' of the system should be under- 
stood in these terms. Even if one waits a viscous time, however, it seems likely that substantial noise could drown the signal. In this sense, conservative equations such as sine-Gordon are not the whole story, particularly for closed systems (e.g. rotating annuli): it is well understood, for example, in convection experiments, that one must wait $\geqslant a$ conductive time scale for patterns to evolve to a steady state (e.g. Busse [6]).

\section{Concluding remarks}

Fluid dynamicists tend to be of the opinion that studies of chaos in simple dynamical systems are not of particular use in the 'real' problems of shear flow and convection (and perhaps other 'nasty' examples), although they have undoubtedly given understanding to a wide variety of other 'weakly' turbulent phenomena in fluids, chemical reactions, population dynamics, etc. Our aim here has been to give some possible reasons why the study of even one simple set of equations may help understanding of physical systems, both by direct application, and by indirect analogy. If any of these ideas eventually bear fruit, then the effort will have been worthwhile.

\section{References}

[1] A. Arneodo., P. Coullet and C. Tresser, J. Stat. Phys. 27 (1982) 171.

[2] T.B. Benjamin and J.E. Feir, J. Fluid Mech. 27 (1967) 417.

[3] D.J. Benney and R. F. Bergeron, Stud. Appl. Math. 48 (1969) 181.

[4] M. Booty, J.D. Gibbon and A.C. Fowler, Phys. Lett. 87A (1982) 261 .

[5] J. Brindley and I.M. Moroz, Phys. Lett. 77A (1980) 441.

[6] F.H. Busse, in Hydrodynamic Instabilities and the Transition to Turbulence, H.L. Swinney and J.P. Gollub, eds. (Springer, Berlin, 1981), p. 97.

[7] P. Collet and J.-P. Eckmann, Iterated Maps on the Interval as Dynamical Systems (Birkhäuser, Boston, 1980).

[8] L.N. Da Costa, E. Knobloch and N.O. Weiss, J. Fluid Mech. 109 (1981) 25 .
[9] A. Davey and H.P.F. Nguyen, J. Fluid Mech. 45 (1971) 701.

[10] R.C. Di Prima and H.L. Swinney, in Hydrodynamic Instabilities and the Transition to Turbulence, H.L. Swinney and J.P. Gollub, eds., (Springer, Berlin, 1981) p. 139.

[11] P.G. Drazin, Quart J. R. Met. Soc. 96 (1970) 667.

[12] P.G. Drazin, J. Fluid Mech. 55 (1972) 577.

[13] J-P. Eckmann, Rev. Mod. Phys. 53 (1981) 643.

[14] M.J. Feigenbaum, J. Stat. Phys. 19 (1978) 25.

[15] P.R. Fenstermacher, H.L. Swinney and J.P. Gollub, J. Fluid Mech. 94 (1979) 103.

[16] A.C. Fowler, IMA J. Appl. Math. 28 (1982) 41.

[17] A.C. Fowler and M.J. McGuinness, A description of the Lorenz attractor at high Prandtl number, Physica 5D (1982) 149

[18] A.C. Fowler and M.J. McGuinness, Hysteresis, period doubling and intermittency at high Prandtl number in the Lorenz equations. Stud. Appl. Math., in press (1983).

[19] A.C. Fowler and M.J. McGuinness, A nonlinear torus at high $r_{1}$ in the complex Lorenz equations. Submitted to SIAM J. Appl. Math. (1983).

[20] A.C. Fowler, J.D. Gibbon and M.J. McGuinness, Physica 4D (1982) 139.

[21] V. Francheschini and C. Tebaldi, J. Stat. Phys. 21 (1979) 707.

[22] S. Fraser and R. Kapral, Phys. Rev. 23A (1981) 3303.

[23] J.D. Gibbon, I.N. James and I.M. Moroz, Proc. R. Soc. Lond. A 367 (1979) 219.

[24] J.D. Gibbon and M.J. McGuinness, Phys. Lett. 77A (1980) 295.

[25] J.D. Gibbon and M.J. McGuinness, Proc. R. Soc. Lond. A 377 (1981) 185 .

[26] J.D. Gibbon and M.J. McGuinness, The real and complex Lorenz equations in rotating fluids and lasers, Physica 5D (1982) 108.

[27] J.D. Gibbon, "The Complex Lorenz Equations", in Chaos and Order in Nature, H. Haken ed. (Springer, Berlin, 1981) (note; some of the figures in this article have been mixed up with those of other articles).

[28] J.P. Gollub and S.V. Benson, J. Fluid Mech. 100 (1980) 449.

[29] W.S.C. Gurney, S.P. Blythe and R.M. Nisbet, Nature 287 (1980) 17.

[30] H. Haken, Encyclopedia of Physics; Light and Matter, vol. 25/2c (Springer, Berlin, 1970).

[31] H. Haken, Phys. Lett. 53A (1973) 77.

[32] J.E. Hart, J. Atmos. Sci. 30 (1973) 1017.

[33] R. Hide and P.J. Mason, Adv. Phys. 24 (1975) 47

[34] F.A. Hopf, D.L. Kaplan, H.M. Gibbs and R.L. Shoemaker, Phys. Rev. A25 (1982) 2172.

[35] L.N. Howard, Int. Cong. Appl. Mech. Munich 1964, H. Görtler, ed. (Springer, Berlin, 1966), p. 1109.

[36] E. Knobloch and M.R.E. Proctor, J. Fluid Mech. 108 (1981) 291.

[37] M.T. Landahl, SIAM J. Appl. Math. 28 (1975) 735.

[38] A. Libchaber and J. Maurer, J. Phys. Lett. 39 (1978) L369. 
[39] A. Libchaber and J. Maurer, J. Phys. 41 (1980) C3, 51.

[40] A. Libchaber, C. Laroche and S. Fauve, J. Phys. Lett. 43 (1982) L211.

[41] E.N. Lorenz, J. Atmos. Sci. 20 (1963) 130.

[42] M.C. Mackey and L. Glass, Science 197 (1977) 287.

[43] P. Manneville and Y. Pomeau, Physica 1D (1980) 219.

[44] S. Maslowe, in Hydrodynamic Instabilities and the Transition to Turbulence, H.L. Swinney and J.P. Gollub, eds. (Springer, Berlin, 1981) p. 181.

[45] J. Maurer and A. Libchaber, J. Phys. Lett. 40 (1979) L419.

[46] J. Maurer and A. Libchaber, J. Phys. Lett 41 (1980) L515.

[47] R.M. May, Nature 261 (1976) 459.

[48] R.M. May, Ann. N.Y. Acad. Sci. 357 (1980) 267.

[49] I.M. Moroz and J. Brindley, Proc. R. Soc. Lond. A 377 (1981) 379.

[50] S.A. Orszag and A.T. Patera, Phys. Rev. Lett. 45 (1980) 989.

[51] E. Ott, Rev. Mod. Phys. 53 (1981) 655.

[52] A.T. Patera and S.A. Orszag, J. Fluid Mech. 112 (1981) 467.

[53] J. Pedlosky, J, Atmos. Sci. 27 (1970) 15.

[54] J. Pedlosky, J. Atmos. Sci. 28 (1971) 587.

[55] J. Pedlosky, J. Atmos. Sci. 29 (1972) 680.

[56] J. Pedlosky, J. Atmos. Sci. 38 (1981) 717.
[57] J. Pedlosky and C. Frenzen, J. Atmos. Sci. 37 (1980) 1177.

[58] O. Reynolds, Phil. Trans. Roy. Soc. 174 (1983) 935.

[59] K.A. Robbins, Proc. Nat. Acad. Sci. USA 73 (1976) 4297.

[60] K.A. Robbins, Math. Proc. Camb. Phil. Soc. 82 (1977) 309.

[61] K.A. Robbins, SIAM J. Appl. Math. 36 (1979) 457.

[62] G.O. Roberts, Geophys. Astrophys. Fluid Dyn. 12 (1979) 235.

[63] S. Rosenblat and S.H. Davis, SIAM J. Appl. Math. 37 (1979) 1.

[64] O.E. Rössler and K. Wegmann, Nature 271 (1978) 89.

[65] D. Ruelle and F. Takens, Commun. Math. Phys. 20 (1971) 167.

[66] T. Shimizu, Physica 97A (1979) 383.

[67] C. Sparrow, The Lorenz Equations: Bifurcations, Chaos, and Strange Attractors. Lecture Notes in Applied Mathematics, Vol. 41 (Springer, Berlin, 1982).

[68] K. Stewartson and J.T. Stuart, J. Fluid Mech. 48 (1971) 529.

[69] C. Tresser P. Coullet and A. Arneodo, J. Phys. Lett. 41 (1980) L243.

[70] D.J. Tritton, D.M. Rayburn and M.A. Forrest, in Mechanisms of Continental Drift and Plate Tectonics, P.A. Davies and S.K. Runcorn, eds. (Academic Press, New York, 1980), p. 267. 